\title{
Research on the Nonlinear Computer Torque control of the MR Damper Based Above-knee Prosthesis
}

\author{
Qiang Fu',a, Chunpeng Pan ${ }^{1}$ and Lei $\mathrm{Xu}^{2}$ \\ ${ }^{1}$ Department of Robot Engineering, Chongqing University of Arts and Sciences, Yongchuan, Chongqing 402160China \\ ${ }^{2}$ Department of Automation, Shanxi University, Taiyuan, Shanxi 030006 China
}

\begin{abstract}
Aiming at the motion track controlling of the semi-active magnetorheological damper based above-knee prosthesis (MRAKP), according to the LaSalle's invariant set theorem, a kind of nonlinear compute torque (NCT) control law for the track controlling of the AKMR, is proposed to promote the robustness and performance of the intelligent above-knee prosthesis. The proposed NCT controller includes the feedforward control and the feedback control. The former one is used to compensate the nonlinear terms in the dynamic model of the MRAKP, such as the Coriolis force, the centripetal force, and the gravity. The feedback control, utilizing a nonlinear PD controller, adaptively adjusts the control gain coefficients and reduces the system error. On these bases, numerical simulations on the MRAKPare carried out to analyze the performance of the proposed NCT controller in ADAMS and simulink. For comparing, the track controlling performance of the PD controller and the CT+PD controller are also presented in the paper. Simulation results indicate that the proposed NCT controller for the MRAKP is able to adaptively adjust the control gain coefficients with lower track error and higher robustness than the conventional PD controller and the $\mathrm{CT}+\mathrm{PD}$ controller.
\end{abstract}

\section{Introduction}

For an amputee, the above-knee prosthesis (AKP) is an irreplaceable rehabilitation appliance due to its incomparable rehabilitation functionality for the patients with trans-femoral amputation. Because of the low energy consumption, high precision and continuous reversible changeable yield stress, magnetorheological (MR) dampers are widely applied for AKPs $[1,2]$.

In the recently decades, there are several researches about the MR damper based above-knee prosthesis (MRAKP) in the world [3-6]. Hugh Herr and Ari Wilkenfeld proposed an intelligent prosthetic knee with a MR damper and the adaptive controlling base on the finite-state machine (FSM) for the prosthetic knee [7]. The proposed control method based on the FSM, which is not need the dynamic of the AKP, is a simple method for the AKP controlling, but is not expert in trajectory tracking. And the torque produced by the proposed controller for the AKP is discontinuous, but the continuous changeable yield stress is precisely the advantage of the MR. Jung-Hoon Kim and Jun-Ho Oh utilized the compute torque (CT) plus proportional derivative (PD) controller to control a MRAKP, and utilized the additional learning control method to reduce the control error[8]. Generally speaking, the close-loop system using the CT+PD controller comprises two channels. Nevertheless, the MRAKP is a strongnonlinearity system in which a small disturbance may cause huge oscillation. So the conventional control methods with fixed parameters are bad at robustness for the MRAKPs in practice [9-13].

In order to improve the motion control for the MRAKP, especially promote the robustness of the controller, a nonlinear control law is proposed according to the theory of the stability analysis. Compared with the conventional PD control law with the fixed control gains, the control gains of the promoted nonlinear control law are adjusted according to the tracking error of the closeloop system.

\section{Model}

\subsection{Dynamical modeling of the AKP}

Figure 1 shows the simplified structural model. As shown in the figure 1, the lower limb is modeled as a two-rigid body with two DOFs in the sagittal plane. The hip and knee joints are simplified as two hinges with variable damping attached on the two ends of the thigh, which is modeled as a link with the mass of $m_{1}$. And the shank and the foot are modeled as an integrated link with the mass of $\mathrm{m}_{2}$ connected with the knee joint. Signals $\theta_{1}$ and $\theta_{2}$ are the anglers of the thigh and the shank relative to direction of the gravity.

According to the Lagrange kinetic, the dynamic of the lower lib can be expressed as [14]

$\overline{{ }^{a} \text { Corresponding author: qfu@cqwu.net }}$ 


$$
\left\{\begin{array}{c}
\tau_{1}-\tau_{2}=M_{11} \ddot{\theta}_{1}+M_{12} \ddot{\theta}_{2}+C_{1}(\theta, \dot{\theta})+G_{1}(\theta) \\
\tau_{2}=M_{21} \ddot{\theta}_{1}+M_{22} \ddot{\theta}_{2}+C_{2}(\theta, \dot{\theta})+G_{2}(\theta)
\end{array}\right.
$$

where $\theta=\left[\begin{array}{ll}\theta_{1} & \theta_{2}\end{array}\right]^{\mathrm{T}}$ denote the generalized state vector, $M=\left[\begin{array}{ll}M_{11} & M_{12} \\ M_{21} & M_{22}\end{array}\right]$ is the inertia matrix, $C=\left[\begin{array}{ll}C_{1} & C_{2}\end{array}\right]^{T}$ is the Coriolis/centripetal vector, $G=\left[\begin{array}{ll}G_{1} & G_{2}\end{array}\right]^{T}$ is the gravity vector, $\tau=\left[\begin{array}{ll}\tau_{1} & \tau_{2}\end{array}\right]^{\mathrm{T}}$ is the generalized torque matrix, in which the $\tau_{1}$ and $\tau_{2}$ is the torque applied to the hip joint and the knee joint respectively.

\subsection{Force model of the MR damper}

In this paper, a MR damper based on the sheer mode is used because of the lower damping coefficient and the simpler force model.

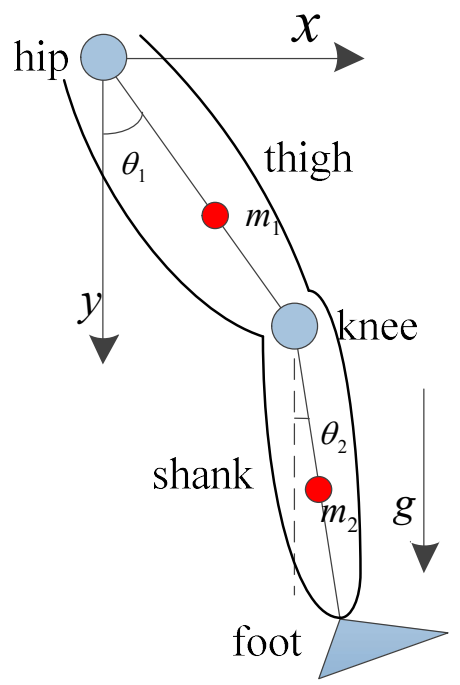

Figure 1. Simplifiedmodel of the lower limb.

The yield stress of the MR damper based on the sheer mode can be expressed as [15]

$$
\mathrm{F}_{\text {field }}=\frac{12 \eta \mathrm{Lw}}{\mathrm{g}} \mathrm{v}_{0}+\mathrm{H}(\mathrm{I}) \mathrm{Lw}
$$

where $F_{\text {field }}$ denotes the yield stress of the MR damper; $\eta$, $\mathrm{L}, \mathrm{w}$, and $\mathrm{g}$ are the structure size of the MR damper; $\mathrm{v}_{0}$ denotes the velocity of the piston of the damper without the drive current; H(I) is the yield stress produced by MR damper with the drive current $I$.

There for, the force model of a MR damper based on the sheer mode is given by

$$
F=F_{\text {spring }}+F_{\text {field }}=K x+\left(\frac{12 \eta L w}{g}+C\right) \dot{x}+H(I) L w
$$

where the $\mathrm{x}, \dot{\mathrm{x}}$ is for the relative displacement and velocity respectively of the piston; $\mathrm{K}, \mathrm{C}$ is the stiffness and damping coefficient respectively. According to the work present in [15], the yield stress H(I) can be expressed by

$$
H(I)=\alpha I^{\beta} \operatorname{sign}(\dot{x})
$$

\subsection{Control system of the AKP}

Figure 2 shows the system diagram of the MRAKP. As shown in figure 2, a MRAKP system includes the gait planner, the controller, and the MRAKP which is composed of the artificial knee joint and the MR damper. The gait planner generates the desired trajectory $\left(\left[\begin{array}{ll}\theta_{\mathrm{d}} & \dot{\theta}_{\mathrm{d}}\end{array}\right]^{\mathrm{T}}\right)$ for the MRAKP according to the practice moving state $\left(\left[\begin{array}{ll}\theta_{1} & \dot{\theta}_{1}\end{array}\right]^{\mathrm{T}}\right)$ of the prosthesis. The controller includes the NCT controller to produce the desired torque $\tau_{\mathrm{N}}$ according to the error [e $\left.\quad \dot{\mathrm{e}}\right]^{\mathrm{T}}$ between the desired trajectory $\left[\begin{array}{ll}\theta_{\mathrm{d}} & \dot{\theta}_{\mathrm{d}}\end{array}\right]^{\mathrm{T}}$ and practice swing angle of the knee joint $\left[\begin{array}{ll}\theta & \dot{\theta}\end{array}\right]^{\mathrm{T}}$, and the inverse MR damper Model to produce the driving current I for the MR damper. The integrated MR damper of the MRAKP produces the damping force $F$, and the artificial knee joint generates the swing angle $\left[\begin{array}{ll}\theta_{1} & \dot{\theta}_{1}\end{array}\right]^{\mathrm{T}}$ driven by damping force of $\mathrm{F}$.

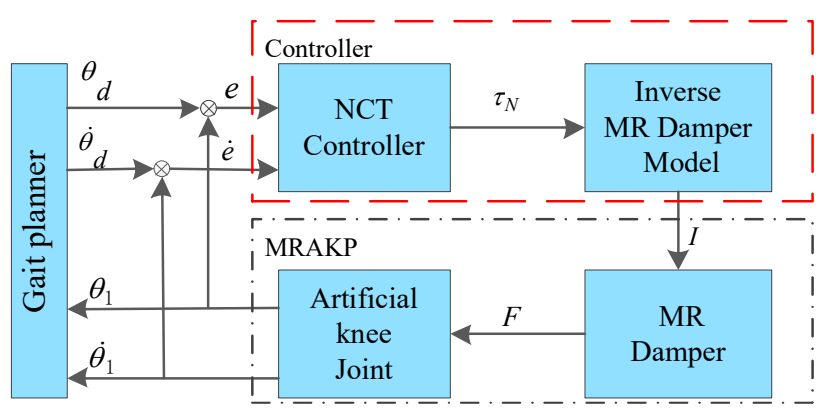

Figure 2. System Diagram of the MRAKP.

\subsection{Non-linear computer torque controller}

The compute torque controller, a kind of linear controller which is widely used for the automatic controlling, is defined as

$$
\begin{gathered}
\tau_{L}=M_{21} \ddot{\theta}_{1}+M_{22}\left(\ddot{\theta}_{d}+k_{p} \mathrm{e}+k_{d} \dot{e}\right)+C_{2}(\theta, \dot{\theta})+ \\
G_{2}(\theta)
\end{gathered}
$$

where the $\theta_{d}$ is the desired trajectory of the knee joint, $k_{p}$ and $\mathrm{k}_{\mathrm{d}}$ are the control gains, $\mathrm{C}_{2}$ and $\mathrm{G}_{2}$ are the Coriolis/centripetal terms and gravity term in the dynamic equation respectively.

The nonlinear computer torque control law, as shown in figure 3 , whose control gains are adjustable according to the track error, is defined as:

$$
\begin{gathered}
\tau_{\mathrm{N}}=\mathrm{M}_{21} \ddot{\theta}_{1}+\mathrm{M}_{22}\left(\ddot{\theta}_{2 \mathrm{~d}}+\mathrm{KP}(\mathrm{e}) \mathrm{e}+\mathrm{KD}(\mathrm{e}) \dot{\mathrm{e}}\right)+ \\
\mathrm{C}_{2}(\theta, \dot{\theta})+\mathrm{G}_{2}(\theta) \\
\left\{\begin{array}{l}
\mathrm{KP}(\mathrm{e})=\mathrm{k}_{\mathrm{p}} \cdot \mathrm{f}(\mathrm{e}) \\
\mathrm{KD}(\mathrm{e})=\mathrm{k}_{\mathrm{d}} \cdot \mathrm{f}(\mathrm{e})
\end{array}\right.
\end{gathered}
$$

where $f(e)$ is the function about the control error, $\operatorname{KP}(e)$ and $\mathrm{KD}(\mathrm{e})$ are the variable control gains.

Substituting equation (6) into (1), the dynamical error of the controlled MRAKP system is given by

$$
\ddot{\mathrm{e}}+\mathrm{KD}(\mathrm{e}) \dot{\mathrm{e}}+\mathrm{KP}(\mathrm{e}) \mathrm{e}=0
$$

The Lyapunov function is defined as

$$
\mathrm{V}=\frac{1}{2} \dot{\mathrm{e}}^{\mathrm{T}} \dot{\mathrm{e}}+\int_{0}^{\mathrm{e}}|\varphi|^{\mathrm{T}} \mathrm{KP}(\varphi) \mathrm{d} \varphi
$$

Deviating equation (9), we have

$$
\dot{\mathrm{V}}=\dot{\mathrm{e}}^{\mathrm{T}} \ddot{\mathrm{e}}+\mathrm{e}^{\mathrm{T}} \mathrm{KP}(\mathrm{e}) \dot{\mathrm{e}}
$$


Substituting equation (8) into (10), we have,

$$
\dot{\mathrm{V}}=-\dot{\mathrm{e}}^{\mathrm{T}} \mathrm{KD}(\dot{\mathrm{e}}) \dot{\mathrm{e}}
$$

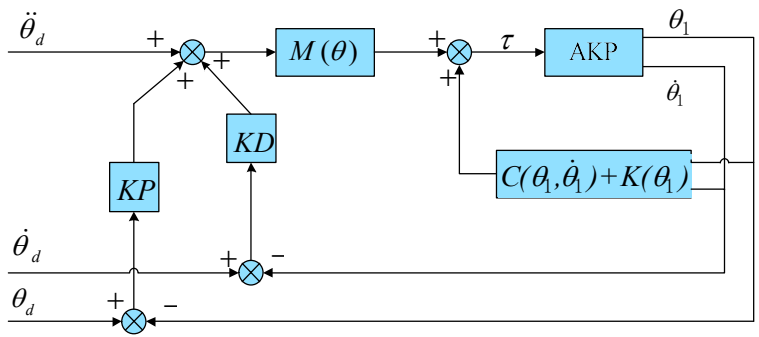

Figure 3. Control diagram of the nonlinear compute torque controller.

Defining $f(e)$ as

$$
\mathrm{f}(\mathrm{e})=\cosh \left(\mathrm{k}_{0} \mathrm{e}\right)
$$

Substituting equation (12) into (7), then $\mathrm{KP}(\mathrm{e})$ and $\mathrm{KD}(\mathrm{e})$ are positive definite, continuous and bounded when $\mathrm{k}_{\mathrm{p}}$ and $\mathrm{k}_{\mathrm{d}}$ are positive.

From (8) to (11), it is shown that $\mathrm{V}>0$, and $\dot{\mathrm{V}} \leq 0$ for any e, and the equilibrium point $\left[\begin{array}{l}\mathrm{e}^{*} \\ \dot{\mathrm{e}}^{*}\end{array}\right]=\left[\begin{array}{l}0 \\ 0\end{array}\right]$ is the largest invariant set of $\Psi=\left\{\left[\begin{array}{l}\mathrm{e} \\ \dot{\mathrm{e}}\end{array}\right] \mid \dot{V}=0\right\}$. So according to the LaSalle's invariant set theory, $\left[\begin{array}{c}\mathrm{e}(\mathrm{t}) \\ \dot{\mathrm{e}}(\mathrm{t})\end{array}\right] \rightarrow\left[\begin{array}{c}\mathrm{e}^{*} \\ \dot{\mathrm{e}}^{*}\end{array}\right]=\left[\begin{array}{l}0 \\ 0\end{array}\right]$ with $\mathrm{t} \rightarrow \infty$. Consequently, if the control law is given by (6), the non-linear feedback system as (8) is globally asymptotical stable.

\subsection{Inverse MR damper model}

Combining equation (3) and (4), the force model of the MR damper is given as

$\mathrm{F}_{\text {damper }}=\mathrm{Kx}+\left(\frac{12 \eta \mathrm{Lw}}{\mathrm{g}}+\mathrm{C}\right) \dot{\mathrm{x}}+\operatorname{Lw} \alpha \operatorname{sign}(\dot{\mathrm{x}}) \mathrm{I}^{\beta}$

where the first and second terms in the right side are uncontrollable terms, and the third term is a controllable term. So the driving current applied on the MR damper can be given by equation (13)

$$
I=\frac{F_{d}-K x-\widehat{C} \dot{x}}{\gamma \operatorname{sign}(\dot{x})}
$$

Because of the character of the MR damper that the direct of the damping force is the direct of the velocity of the piston [15], as shown in figure 4 , so that $\dot{x}>0$ due that only the extend of the MRAKP in the swing phase are controllable. As a result, equation (14) can be rewrite as

$$
\mathrm{I}=\frac{\tau_{\mathrm{N}} \cdot \psi\left(\theta_{1}, \theta_{2}\right)-\mathrm{Kx}-\widehat{\mathrm{C}} \dot{\mathrm{x}}}{\gamma}
$$

where $\widehat{\mathrm{C}}\left(=\frac{12 \eta \mathrm{Lw}}{\mathrm{g}}+\mathrm{C}\right)$ and $\gamma(=\mathrm{Lw} \alpha)$ are constants, $\mathrm{x}$ $\left(=\Phi\left(\theta_{1}, \theta_{2}\right)\right)$ denotes the displace of the piston of the damper, and $\psi\left(\theta_{1}, \theta_{2}\right)$ is the arm of force.

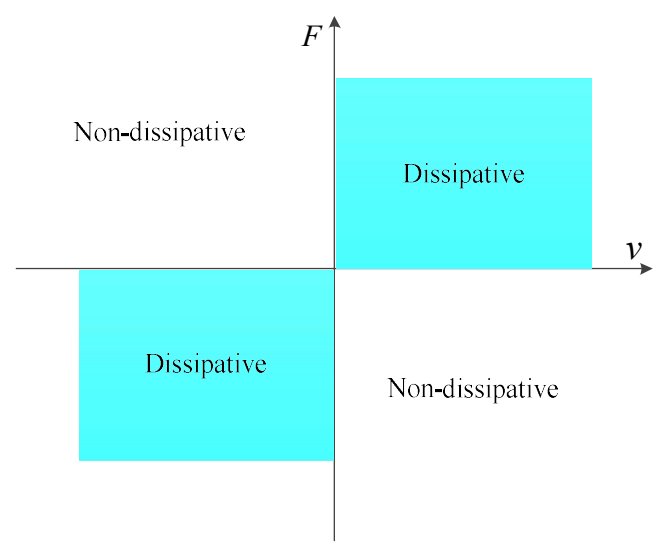

Figure 4. Character of the yield stress of the semi-active MR damper [15].

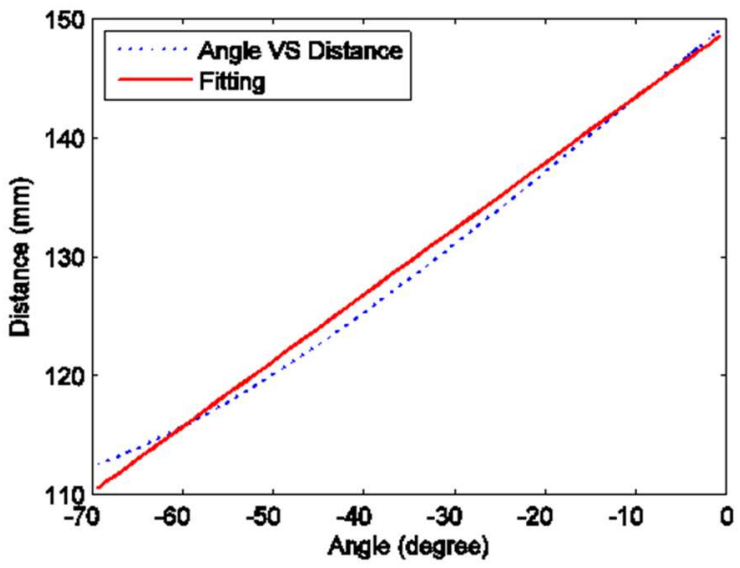

Figure 5. Relation between the distance of the piston and the knee angle

Analyzing the relation between the stroke of the MR damper and the knee angle, as shown in figure 5, the stroke of the MR damper can be approximately consider as the linear function of the knee angle and expressed as

$$
\mathrm{x}=0.5507 \cdot\left(\frac{\pi}{2}-\theta_{2}+\theta_{1}\right)+148.3
$$

\section{Simulation and analysis}

In order to analyze the performance and robustness of the promoted non-linear computer torque controller of the MRAKP, the co-simulation model of the MRAKP are established in ADAMS and simulink shown in figure 6.

Figure 7(a) and 7(b) show the simulated controlled angles and control errors of the MRAKP controlled by the $\mathrm{PD}, \mathrm{CT}+\mathrm{PD}$ and proposed NCT controllers. The results shown in figure 7 illustrate that the swing angles of the MRAKP with controllers match to the reference angle more accurately thanthe MRAKP without a controller does. However, the tracking error of the PD controller, which is a linear controller, is larger than the errors of others. Compared with the PD controller, the $\mathrm{CT}+\mathrm{PD}$ controller is more effective because of the feedforward control for compensating the non-linear terms in the dynamic model of the MRAKP. What the most worth for mention is that, the proposed NCT controller has the lowest control error compared with other controllers 
because of adaptive variable control gains, which are shown in figure 8.

The result of the co-simulation of the AKP with variable frequency is shown in figure 9. As figure 9 shows, the MRAKP walked at three different frequencies, $0.5 \mathrm{HZ}, 1.0 \mathrm{HZ}$ and $2 \mathrm{HZ}$ (Natural frequency of normal human walking is about $1.5 \mathrm{HZ}[16])$. The result indicates that the tracking error of the conventional controller (PD, $\mathrm{CT}+\mathrm{PD}$ ) and proposed NCT controller larger and larger with the increasement of the frequency, nevertheless, the increasement of the NCT controller is smaller than those of others. As shown in figure 9-b, the tracking error of NCT controller is $60 \%$ lower than that of PD controller, and just half of the track error of the $\mathrm{CT}+\mathrm{PD}$ controller. In conclusion, the proposed NCT controller is more precise and robust if the frequency of motion of AKP changes widely.

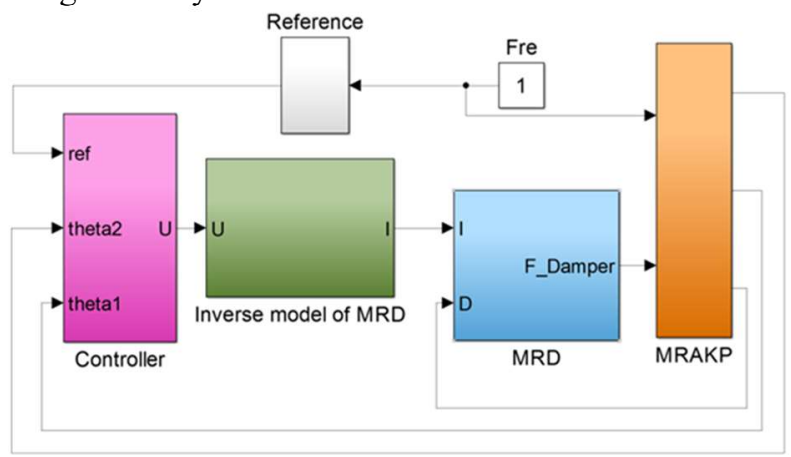

Figure 6. Co-simulation model of the MRAKP.

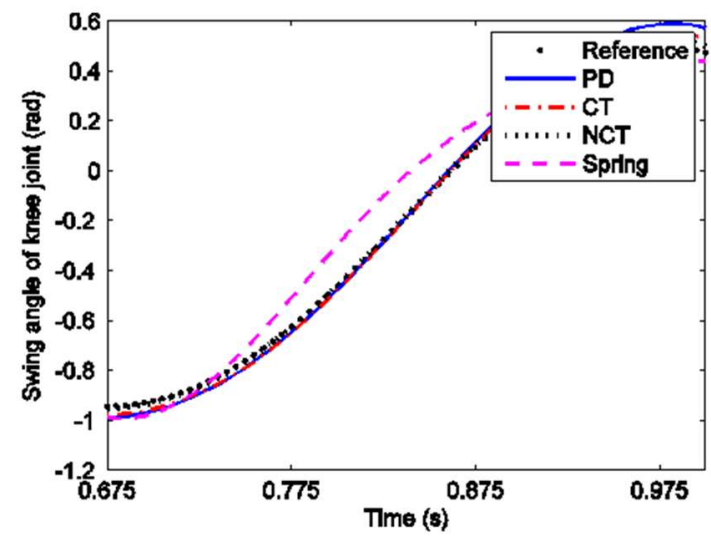

(a)

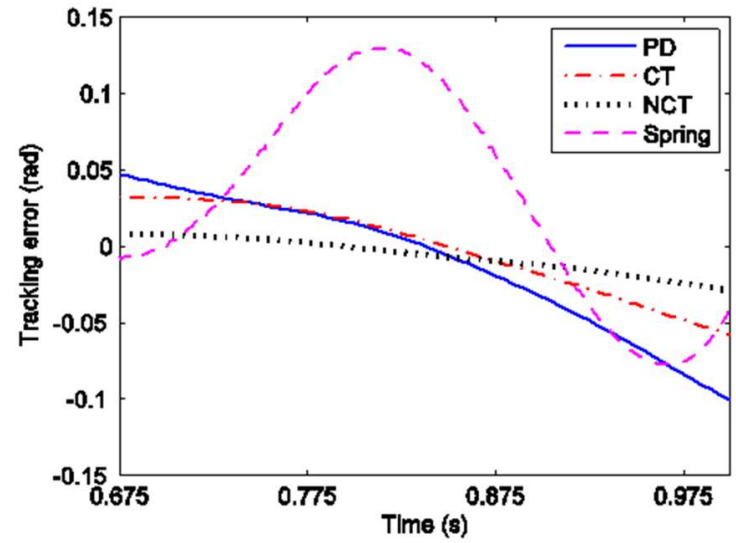

(b)

Figure 7. Tracking control with the invariable frequency (a) knee angle tracking (b) tracking error.

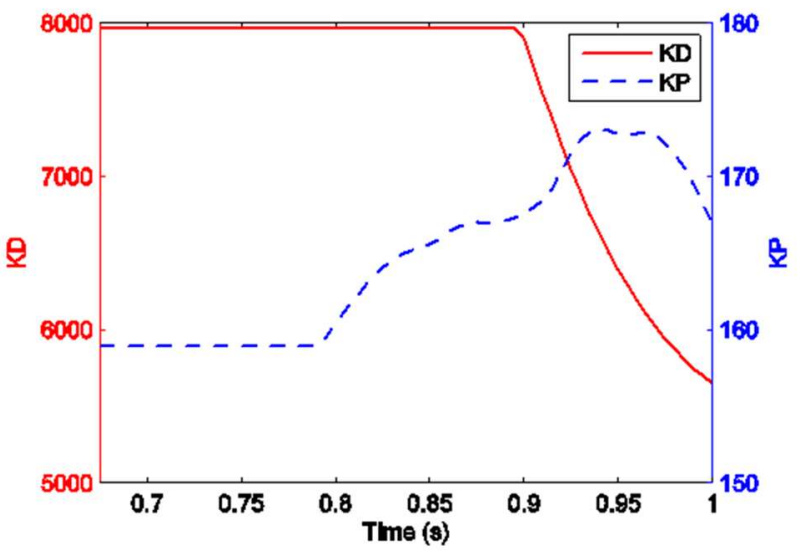

Figure 8. Control gain of the nonlinear compute torque controller.

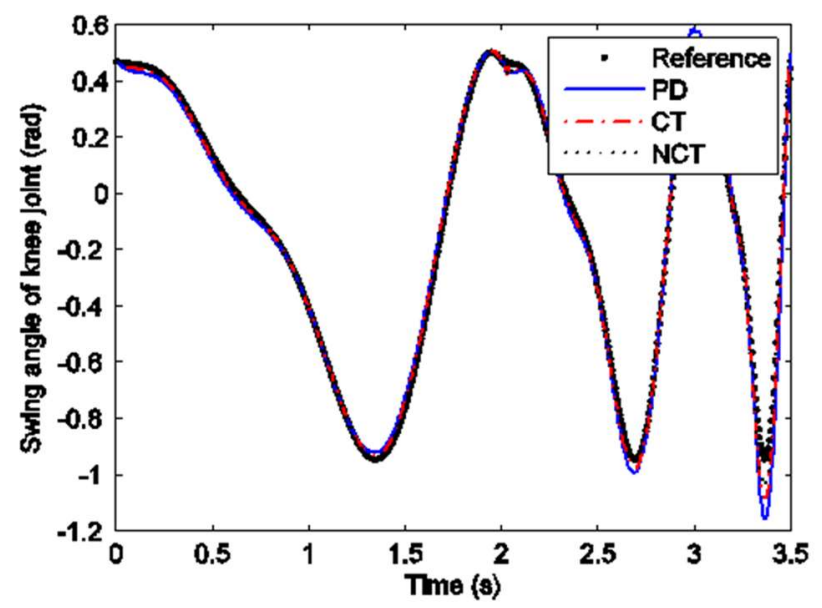

(a)

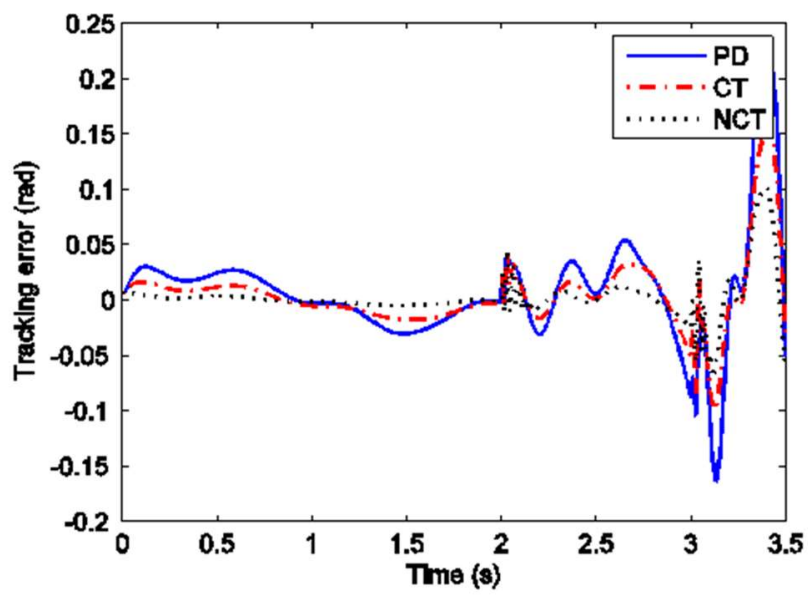

(b)

Figure 9. Tracking control with the variable frequency (a) knee angle tracking (b) tracking error.

\section{Discussion and conclusion}

In order to improve the motion control for the MRAKP, especially promote the robustness of the controller, a nonlinear control law is proposed according to the theory of the stability analysis. Compared with the conventional PD control law with the fixed control gains, the control gains of the promoted nonlinear control law are adjusted according to the tracking error of the close-loop system. 
On these bases, numerical simulations on the MRAKPare carried out to analyze the performance of the proposed NCT controller in ADAMS and simulink. For comparing, the track controlling performance of the PD controller and the CT+PD controller are also presented in the paper. Simulation results indicate that the proposed non-linear compute torque controller is more accuracy than the conventional PD controller and CT + PD controller. And the NCT controller is more robust due to the adaptive adjustable control gains. Furthermore, according to the LaSalle's invariable set theory, the NCT is a kind of globally asymptotical stable controller.

\section{Acknowledgements}

This work has been supported by Scientific and Technological Research Program of Chongqing Municipal Education Commission (Project No. KJQN201801328), Scientific and Technological Research Program of Chongqing University of Arts and Sciences (Project No. R2018JD03), and Scientific and Technological Research Program of Chongqing University of Arts and Sciences (Project No. R2018JD05).

\section{References}

1. Calson J D, Catanzarite D M, and Clari K A S, Commercial magneto-rheological fluid devices, International Journal of Modern Physics B, Vol.10, 1996: 2857-2865.

2. Hsu $\mathrm{H}$ and Viejo A, Magnetorheological fluid compositions and prosthetic knees utilizing same: US, 0197051 A1, 2006-09-7.

3. Park J, Yoon G H, Kang J W, and Choi S B, Design and control of a prosthetic leg for above-knee amputees operated in semiactive and active modes, Smart Materials and Structures, 2016, Vol. 25, No. 8, 275-296

4. $\mathrm{Xu} \mathrm{L}$, Wang D H, Fu Q, Yuan G, Hu L Z, A novel four-bar linkage prosthetic knee based on magnetorheological effect: principle, structure, simulation and control, Smart Materials and Structures, 2016,25(11), 115007 (12pp).

5. Guo $\mathrm{H} \mathrm{T}$ and Liao $\mathrm{W} H$, Design and control of multifunctional magnetorheological actuators for assistive knee braces, Proceedings of Active and Passive Smart Structures and Integrated Systems 2010, San Diego, CA, 2010:1-11

6. Xie H L, Liang Z Z, Li F, and Guo L X, The knee joint design and control of above-knee intelligent bionic leg based on magneto-rheological damper, International journal of Automation and Computing, 2010, Vol. 7: 277-282

7. Herr $\mathrm{H}$ and Wilkenfeld A, User-adaptive control of a magnetorheological prosthetic knee, Industrial Robot, 2003, Vol. 30: 42-55

8. Kim J H and Oh J H, Development of an above knee prosthesis using MR damper and leg simulator, Proceedings of the 2001 IEEE International Conference on Robotics \& Automation. Seoul, Korea, 2001: 3686-3691

9. Popovic D B, Out Space Tracking Control for Above-Knee Prosthesis, IEEE Transactions on Biomedical Engineering, Vol. 40, 1993: 549-557

10. Hiemenz G J, Hu W, and Wereley N M, Semi-Active Magnetorheological Helicopter Crew Seat Suspension for Vibration Isolation, Journal of Aircraft, Vol. 45, 2008: 945-953

11. Xu Y, Hollerbach J M, and Ma D, A nonlinear PD controller for force and contact transient control, IEEE Transactions on Control System Mag, Vol. 15, 1995:15-21

12. Shang W W, Cong S, and Zhang Y X, Nonlinear friction compensation of a 2-DOF planar parallel manipulator, Mechatronics, Vol. 18, 2008:340-346

13. Kelly R, and Ricardo C, A class of nonlinear PDtype controller for robot manipulator, J Robotic System, Vol. 13, 1996:945-950

14. Fu Q, Wang D H, Xu L, and Yuan G, A magnetorheological damper based prosthetic knee (MRPK) and the sliding mode tracking control method for the MRPK based lower-limb prosthesis, Smart Materials and Structures, 2017,26(6), 045030(14pp).

15. Tsang H H, Su R K L, and Chandler A M, Simplified inverse dynamics models for MR fluid dampers. Engineering Structures, Vol. 28, 2006: 327-341

16. Murray M P, Drought A B, and Kory R C, Walking patterns of normal men, The Journal of Bone and Joint Surgery, Vol. 46, 1964: 335-360 